Cell Research (2003); 13(4):275-283

http://www.cell-research.com

\title{
Mitosin/CENP-F is a conserved kinetochore protein subjected to cytoplasmic dynein-mediated poleward transport
}

\author{
Zhen Ye YANG, Jing GUO, Ning LI, Min QIAN, Sheng Nian WANG, Xue Liang ZHU* \\ Laboratory of Molecular Cell Biology, Institute of Biochemistry and Cell Biology, Shanghai Institutes for Biological \\ Sciences, Chinese Academy of Sciences, 320 Yueyang Road, Shanghai 200031, China
}

\begin{abstract}
AABSTRACT
Mitosin/CENP-F is a human nuclear protein transiently associated with the outer kinetochore plate in M phase and is involved in M phase progression. LEK1 and CMF1, which are its murine and chicken orthologs, however, are implicated in muscle differentiation and reportedly not distributed at kinetochores. We therefore conducted several assays to clarify this issue. The typical centromere staining patterns were observed in mitotic cells from both human primary culture and murine, canine, and mink cell lines. A C-terminal portion of LEK1 also conferred centromere localization. Our analysis further suggests conserved kinetochore localization of mammalian mitosin orthologs. Moreover, mitosin was associated preferentially with kinetochores of unaligned chromosomes. It was also constantly transported from kinetochores to spindle poles by cytoplasmic dynein. These properties resemble those of other kinetochore proteins important for the spindle checkpoint, thus implying a role of mitosin in this checkpoint. Therefore, mitosin family may serve as multifunctional proteins involved in both mitosis and differentiation.
\end{abstract}

Key words: mitosin, LEK1, CENP-F, kinetochore.

\section{INTRODUCTION}

Mitosin/CENP-F is a $350-\mathrm{kD}$ kinetochore protein [1-3]. Electron microscopy reveals that mitosin/CENP$\mathrm{F}$ is located at the outer kinetochore plate[2, 4]. In primate cell lines studied to date, mitosin is expressed in a cell-cycle dependent manner. The protein level is low in G1 phase, but increases sharply in S phase as a nuclear protein. Following the progression of $\mathrm{M}$ phase, mitosin is hyperphosphorylated and exhibits localization at the kinetochores, spindle poles, and midbody $[1,3,5]$. It is subjected to rapid degradation at the end of mitosis[1]. In addition, mitosin/CENP-F is farnesylated at the C-terminal CAAX motif, a modifi-

\footnotetext{
* Correspondence: Prof. Xue Liang ZHU

Institute of Biochemistry and Cell Biology, 320 Yueyang Road, Shanghai 200031, China Tel: 0086-21-54921406 Fax: 0086-2154921011 E-mail: xlzhu@sibs.ac.cn

Received April-24-2003 Revised June-24-2003 Accepted June-272003
}

cation which<directs its localization at the nuclear envelope[6, 7].Overexpression of the C-terminal portion of mitosin capable of kinetochore localization delays progression through $\mathrm{G} 2 / \mathrm{M}$ in farnesylation-dependent way[1, 7]. These results suggest roles of mitosin/CENP-F in M phase progression, especially chromosome segregation.

Orthologs of mitosin, including avian CMF1 and murine LEK1, have recently been characterized[811]. These proteins share overall similarities ranging from $60 \%$ to $80 \%$ and are characteristic of multiple hydrophobic zippers. However, according to reports to date, they appear to differ in expression patterns and even functions. Distribution of CMF1 appears to be restricted to myogenic tissues. It is highly expressed in differentiating chicken heart from stages 9 to 18; after this, the expression level drop dramatically $[8,10]$. Subcellular localization of CMF1 is variable. It exhibits cytoplasmic localization throughout the differen- 
tiation stages of cardiac myocytes[10]. In skeletal myocyte, however, CMF1 first appears as a nuclear protein in early myoblasts, then as a cytoplasmic protein in differentiating myoblasts[11]. In contrast to presumed functions of mitosin/CENP-F as a general $\mathrm{M}$ phase regulator, $\mathrm{CMF} 1$ is involved in induction and/ or accumulation of myosin in differentiating myocytes $[8,11]$.

LEK1 also implicated in muscle differentiation[9]. It is ubiquitously expressed in early mouse embryo. However, there is a general cessation of expression throughout the embryo as cell division subsides. LEK1 is not detected in adult proliferative cells, for instance, skin and intestinal epithelia. However, it is highly expressed in the nuclei of all interphase $\mathrm{C} 2 \mathrm{C} 12$ cells before and after induction of myotube formation. Similar to CMF1, LEK1 is not detected at the kinetochore in mitotic $\mathrm{C} 2 \mathrm{C} 12$ cells[9], leading to a speculation that the kinetochore localization of mitosin/CENP-F is merely due to deregulations in transformed cells[9].

We therefore performed a variety of assays to clarify some of the discrepancies. Our results suggest that the kinetochore distribution is an intrinsic property of all mammalian mitosin and imply involvement of mitosin/CENP-F in the spindle checkpoint, a mechanism that guarantees proper timing of anaphase onset $[12,13]$. Therefore, the mitosin/CENP-F family proteins may exert multiple functions in growth and differentiation.

\section{MATERIALS AND METHODS}

\section{Antibody production}

Fusion protein MBP-10C, containing amino acid 2,484 to 3,113 of mitosin, was expressed and purified as described previously[1]. Purified protein was used as immunogen to immunize chicken. a10C IgY was purified from egg yolk by polyethylene glycol (PEG) precipitation method[14]. The specificity of a10C antibody was tested by immunoblotting and immunofluorescence.

\section{Plasmid construction}

cDNA encoding the C-terminus of murine LEK1 nucleotides (4, 714-7,492)[9] was obtained by RT-PCR from mouse embryos 12 days post gestation. The PCR products were cloned into pUHD30F, a vector containing a FLAG-coding sequence driven by the tetracycline-response promoter[5]. The sequence was confirmed by sequencing using GenBank accession AF194970 as the reference sequence.

\section{Cell culture and transfection}

Human lymphokine-activated killer (LAK) cells were maintained in RPMI medium 1640 (GIBCO); other cell lines were maintained in Dulbecco's modified Eagle's medium (DMEM, GIBCO) supplemented with $10 \%$ calf serum (Sijiqing company, Hangzhou, China) in an atmosphere containing $5 \% \mathrm{CO}_{2}$. Primary culture of human umbilical vein endothelial cells (HUVEC) were freshly prepared[15]. Transfection was performed using the calcium phosphate method as described[1].

\section{ATP inhibitor assay}

The ATP inhibitor assay was performed as described[16,17]. Briefly, cells were rinsed twice with phosphate-buffered saline to remove culture medium and soaked in saline alone or saline plus 5 $\mathrm{m} M$ Na-azide (Sigma) and $1 \mathrm{mM}$ 2-deoxyglucose (Fluka) at $37^{\circ} \mathrm{C}$ for 30 min prior to fixation with methanol for IIF staining.

\section{Immunofluorescence microscopy and immunoblotting}

Indirect immunofluorescence (IIF) labeling and chromosome spreads were performed as previously described[1,18]. Nuclear DNA was stained with DAPI (4',6'-diamidino-2-phenylindole). Anti-FLAG M2 mAb (Sigma), anti-mitosin mAb (Transduction Laboratories, KY) were used at 1:1000, 1:200 dilution. The appropriate secondary antibodies were diluted to 1:100. Images were collected by confocal microscopy (ACAS Ultima) or using cool CCD SPOT II (Diagnostic instruments, Inc) on Olympus BX51 microscope and were reorganized using Adobe Photoshop software.

For immunoblotting, cells were lysed directly in SDS-Loading buffer. Samples were subjected to SDS-PAGE prior to immunoblotting. 3-12\% gradient SDS-PAGE was used for full-length mitosin.

\section{PCR and cloning for mitosin gene fragments}

To clone mammalian mitosin orthologs, fresh liver samples were cut into pieces and digested with proteinase $\mathrm{K}(100 \mu \mathrm{g} / \mathrm{ml}$; Promega) at $55^{\circ} \mathrm{C}$ overnight in lysis buffer $(50 \mu M$ Tris-HCl, $\mathrm{pH}$ 8.0, $0.1 M$ EDTA, $0.5 \%$ SDS). Followed by phenol/chloroform extraction and ethanol precipitation. Degenerate PCR primers MA (5' GARGCYGATGAAAAGAAGCAGCT 3'; Y = C or T, R = A or G) and MB (5' CTCATTKAGCTCYTTTAAYTG 3'; K = G or T) were based on two conserved regions within the internal repeats of human mitosin ("EADEKKQL" between amino acids 2,128-2,135 and 2,310-1,317, and "QLKELNE" between amino acids 2,263-2,269 and 2,435-2,441). Wax-mediated Hot Start PCR [19] was performed using genomic DNA as template and Ex Taq from TaKaRa Biotechnology Co. (Dalian, China). PCR products migrated at positions equivalent to about $0.5 \mathrm{~Kb}$ and $0.9 \mathrm{~Kb}$ were purified and subcloned into Tvector (Promega). Southern hybridization to plasmid preparations was performed using radioactively-labeled human mitosin cDNA fragment as a probe (nucleotides 6,349-7,533)[19]. Positive plasmids were then subjected to restriction analysis and sequencing. Desired ones were then fully sequenced.

Sequences were analyzed using DNASTAR software (DNASTAR 
Inc., Madison). Divergence of DNA sequences was calculated as percentage of replacement mutations, i. e. mutations which resulted in nonsynonymous codon changes[20].

\section{RESULTS}

Kinetochore localization of mitosin in primary cells

To study the subcellular localization of mitosin homologs in non-primate cell lines, antibodies with broader reactivity are required. Since our anti-mitosin antibodies for previous studies recognize only primate mitosin[1,5], we generated a new polyclonal antibody in chicken using the same immunogen as for the production of $\alpha 10 \mathrm{C}$ antibody[1]. As shown in Fig 1A, purified a10C IgY (i.e., yolk IgG) specifically recognized both the unphosphorylated form of mitosin in interphase HEK293 cells (lane 1) and hyperphosphorylated mitosin existing only in mitotic cells (lane 2, upper band).

$\alpha 10 \mathrm{C}$ IgY also generated IIF staining typical of mitosin (Fig 1B). In CV1 cells, mitosin is highly expressed in cells in S phase but not G1 (Fig 1B, panels $1-2)$; it is concentrated on kinetochores in addition to some distribution in cytoplasm during $\mathrm{M}$ phase (panels 3-4)[1]. Similar patterns were also observed in other primate cell lines, including hepatoma, SMMC-7721 and human embryonic kidney HEK293 (data not shown). The chicken antibody a10C IgY is therefore specific to mitosin.

To test if distribution of mitosin at the kinetochore is only restricted in immortalized cell lines as suspected

$\triangleright$ Fig 1. Kinetochore localization of mitosin in primary cells. Anti-mitosin antibody a 10C IgY (yolk IgG) was purified from yolks of chicken eggs. For IIF, cells growing on coverslips were fixed in cold methanol prior to staining with a10C IgY. Nuclear DNA was labeled with DAPI. Scale bar, $10 \mathrm{~mm}$. (A) a10C IgY recognizes mitosin on Western blot. HEK293 cells were blocked at $\mathrm{G} 1 / \mathrm{S}$ with thymidine or $\mathrm{M}$ phase with nocodazole. Cell lysate was subjected to 3-12\% gradient SDS-PAGE and Western blotting using a10C IgY (lanes 1-2) and rabbit a10Stu antiserum (lanes 3-4)[5]. The upper band is the hyperphosphorylated mitosin detected only in mitotic cells[1]. (B) a 10C IgY stains typical patterns of mitosin in CV1 cells. Panels 1-2, interphase cells. Arrow and arrowhead indicate G1 and S/G2 cells, respectively, judged by expression levels of mitosin[1]. Panels $3-4$, a mitotic cell. Bright fluorescence dots at the DNA region are the centromere staining of mitosin[1]. (C) Centromere localization of mitosin in primary cells. Mitotic cells are indicated by arrows.
[9], we examined two human primary cultures, umbilical vein endothelial (HUVE) cells (Fig 1C, panels 1-2) and lymphokine-activated killer (LAK) cells (Fig 1C, panels 3-4). In mitotic cells of both cell lines, the antimitosin IgY labeled bright punctate foci typical of centromere/kinetochore localization of mitosin.

Kinetochore localization of mitosin orthologs in nonprimate mammalian cells
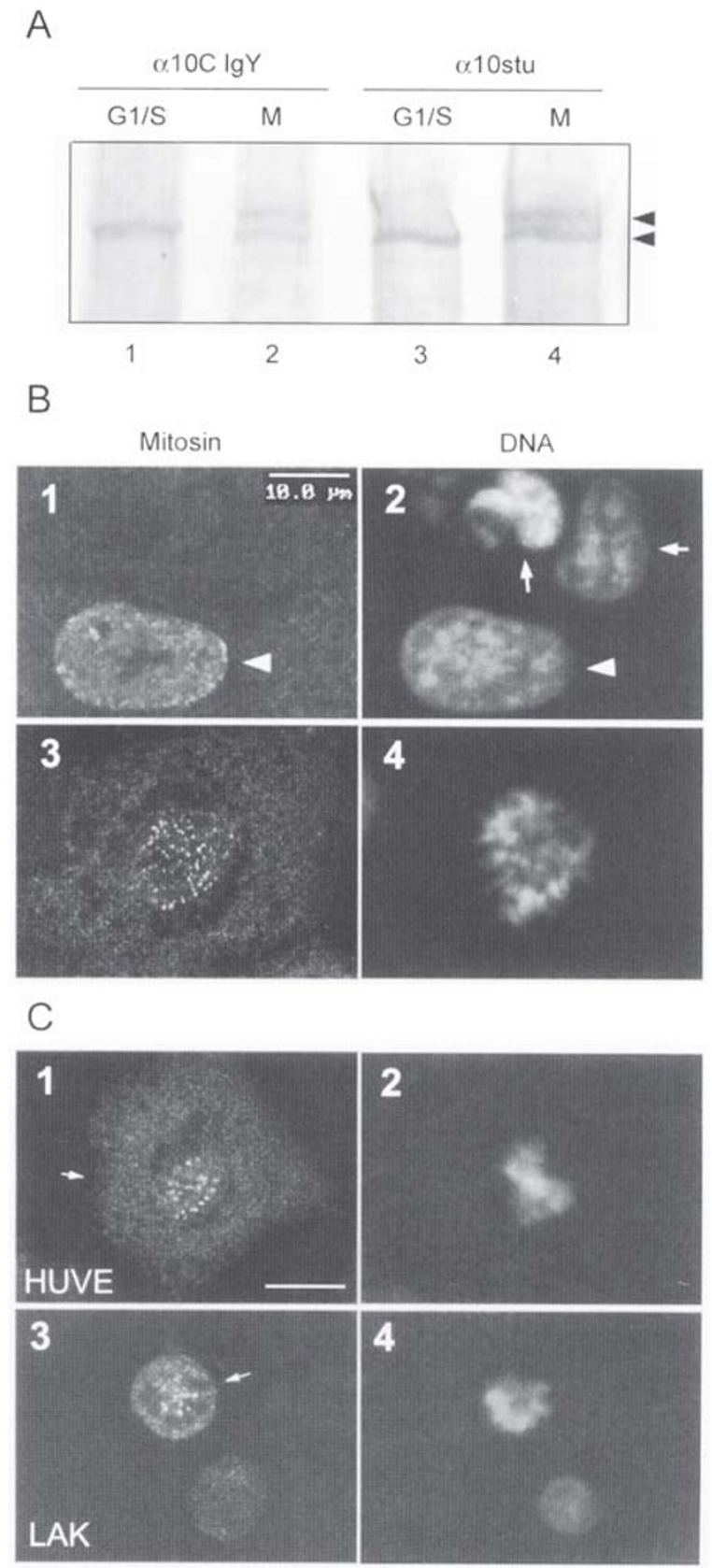
A

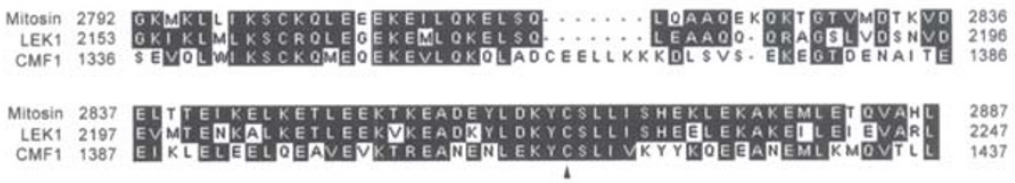

B
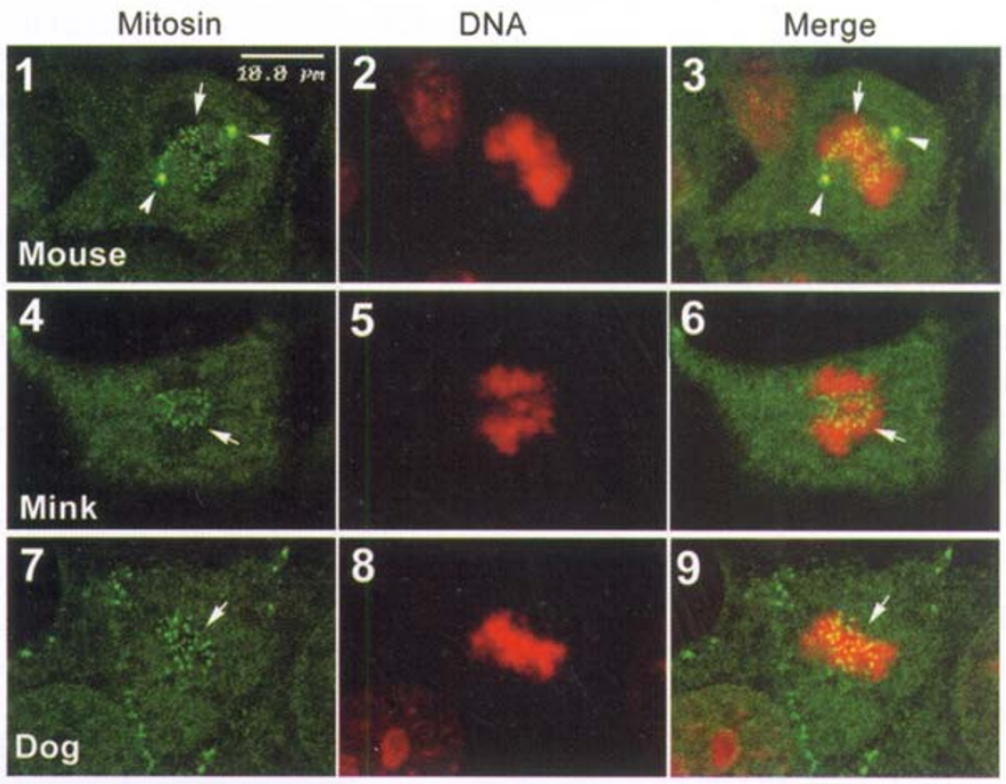

C

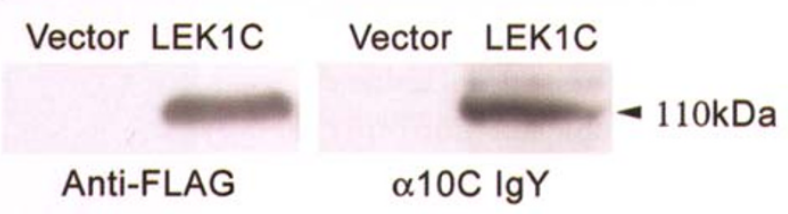

D
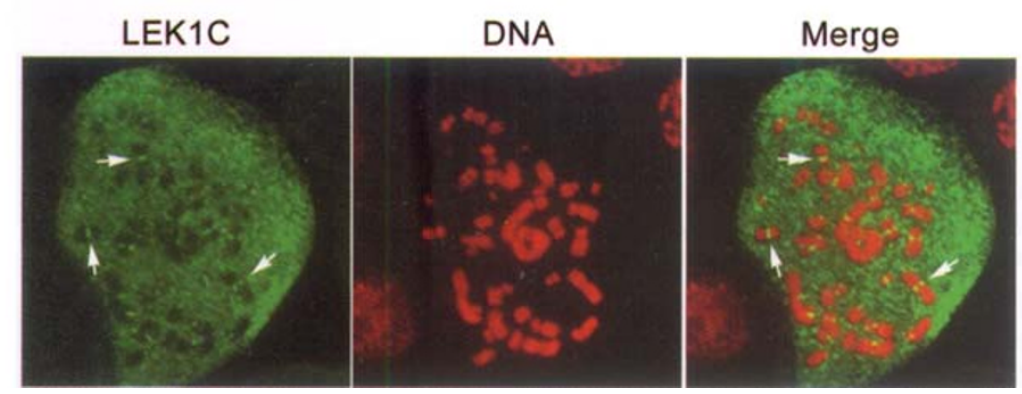

Fig 2. Kinetochore localization of mammalian mitosin orthologs. Scale bar $=10 \mu \mathrm{m}$ (A) Conservation of the core region of mitosin in LEK1 and CMF1. The arrowhead indicates cysteine-2864 critical for activity of the core region. Residues D/ E, L/I/V, N/Q, R/K, or S/T are considered similar. (B) Three cell lines from mouse, mink, and dog were fixed by cold methanol and subjected to IIF using $\alpha 10 \mathrm{C}$ IgY (green). Nuclear DNA was stained by DAPI (red). Images were digitized by confocal microscopy. Areas with centromeres staining are marked by arrows. In addition to centromeres, the antibody also decorated the spindle poles (arrow heads) in NIH3T3 (panels 1-3). (C) $\alpha 10 \mathrm{C} \mathrm{IgY} \mathrm{recognizes} \mathrm{LEK.} \mathrm{A} \mathrm{C-terminal} \mathrm{portion} \mathrm{of} \mathrm{LEK1}$ (LEK1C) was tagged with FLAG and expressed in HEK293T cells. Cell lysates were subjected to SDS-PAGE and immunoblotting with indicated antibodies. Cells transfected with vector alone served as control. ( D) Localization of LEK1C to centromeres. CHO cells expressing FLAG-LEK1C were enriched for M phase population prior to chromosome spread. IIF was then performed with anti-FLAG antibody (green). Three chromosomes with typical centromere staining of LEK1C are indicated by arrows in the merged image. 
LEK1 is reportedly not a centromere protein[9]. We have previously shown that two sequence regions are critical for stable binding of mitosin to centromeres: the core region between residues $2,792-2,887$ and the internal repeat from residues 2,111 to 2,470 [18]. Both LEK1 and CMF1 contain regions resembling the core region of mitosin (Fig 2A).Cysteine-2864 essential for the activity of the core region of mitosin[18] is also conserved (Fig 2A). LEK1 also has a similar repeat region, although CMF1 contains only one such repeat unit[9,10 ]. To clarify the discrepancy, we examined three mammalian cell lines originated from mouse (NIH3T3), dog (MDCK), and mink (Mv $1 \mathrm{Lu})$. IIF staining using $\alpha 10 \mathrm{C}$ IgY revealed typical centromere staining patterns in the mitotic cells (Fig $2 \mathrm{~B}$ ). These results suggest that mitosin orthologs in these species are kinetochore proteins.

To corroborate the observations, we further checked if the centromere localization detected in mouse cells (Fig 2B, panels 1-3) was due to LEK1. We cloned LEK1 cDNA from mouse embryos 12 days post gestation to express FLAG-tagged LEK1C, a polypeptide containing the C-terminal 903 amino acids. The corresponding region of human mitosin exhibits typical kinetochore localization[18]. FLAG-LEK1C migrated as a protein of $\sim 110 \mathrm{kD}$ as revealed by immunoblotting using anti-FLAG mAb ((Fig 2C). It was also recognized by the anti-mitosin antibody $\alpha 10 \mathrm{C} \operatorname{IgY}$ ((Fig 2C). Moreover, FLAG-LEK1C was a nuclear protein in interphase and exhibited patterns resembling centromere localizing in mitotic cells when expressed in HEK293T, HeLa, or CV1 cells (data not shown). Chromosome spread of mitotic $\mathrm{CHO}$ cells further confirmed that, similar to mitosin, a fraction of LEK1C was indeed localized at centromeres (Fig 2D, panels 1-3).

\section{Preferred binding of mitosin to unaligned chromo- somes}

The spindle checkpoint functions by sensing the extent of bilateral microtubule attachment to and/or tension across each pair of sister kinetochores. To date, proteins involved in the spindle checkpoint either directly or indirectly exhibit reduced binding to kinetochores after microtubule attachment, a property consistent with their "sensory" functions on completion of bipolar microtubule attachment[12,21,22]. Among them, kinetochore-bound Mad2, CLIP-170, Rod,
ZW10, cytoplasmic dynein, and dynactin are significantly depleted upon microtubule attachment, while Bub1, Bub3, BubR1, and CENP-E are moderately attenuated; in contrast, those which are believed to be structural proteins, for instance, CENP-B and
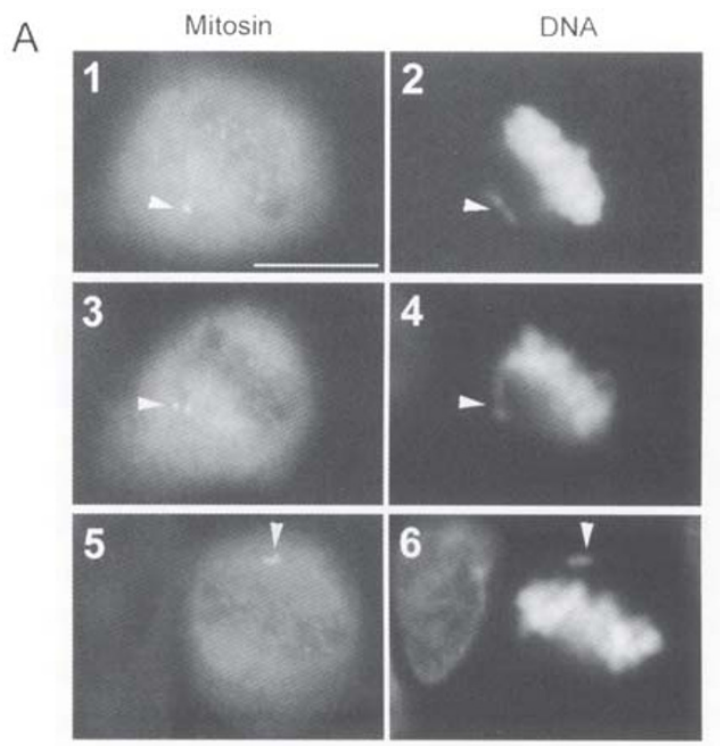

B
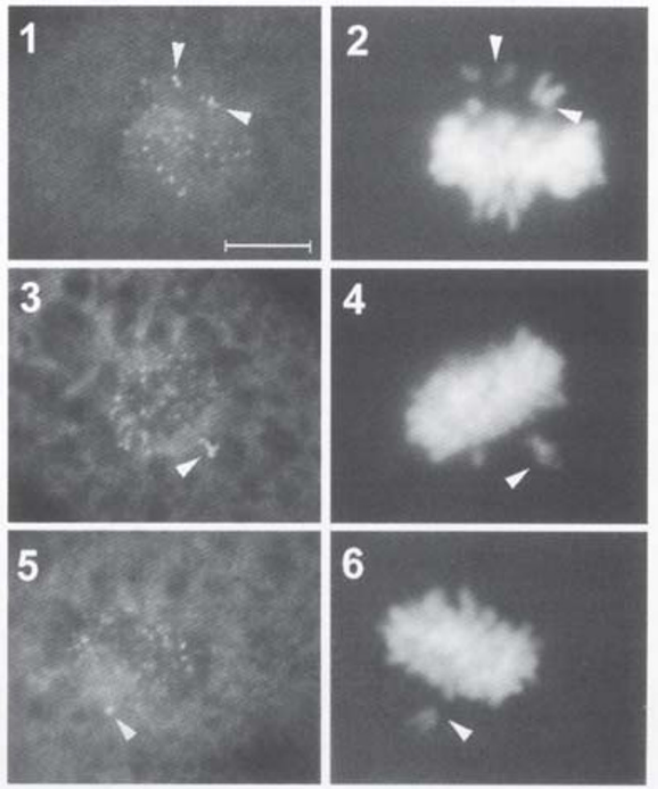

Fig 3. Increased binding of mitosin to unaligned chromosomes. Three typical mitotic HEK293 cells (A) and three CV1 cells (B) with unaligned or misaligned chromosomes (indicated by arrowheads) are shown. Kinetochore staining of mitosin was visualized using anti-mitosin $\mathrm{mAb}$. Bar $=10 \mu \mathrm{m}$. 
CENP-C, remain unchanged[[12, 16, 17, 21-24]. ]. It has been noticedthat the immunofluorescence of mitosin on kinetochores peaks in prophase and prometaphase, and declines after metaphase[1,3]. Such a difference might also correspond to microtubule attachment to the kinetochores. When examining unaligned chromosomes in HEK293 cells, we confirmed that mitosin indeed showed elevated binding to their kinetochores. Of 30 such chromosomes, 23 showed stronger centromere immunofluorescence of mitosin than those aligned at the metaphase plates (Fig 3A). Such difference was not due to location of centromeres in different focal plains of the microscope. Similar result was also obtained in CV1 cells (Fig 3B). Kinetochores on unaligned chromosomes are usually not attached by bipolar microtubules. Increased binding to such kinetochores implies that mitosin might function in the spindle checkpoint.

Transport of mitosin from kinetochores to spindle poles by cytoplasmic dynein during mitosis

In PtK1 cells, proteins involved in the spindle checkpoint, for instance, Mad2, BublR1, CENP-E, and 3F3/2 phospho-epitope, are transported from kinetochores to spindle poles by the microtubule-based motor, cytoplasmic dynein, while structural proteins are not transported[17]. This process also contributes to inactivation of the spindle checkpoint[16,17]. The transport is easily observed when cellular ATP production is suppressed by azide, under which condition these proteins are depleted from centromeres and accumulated at spindle poles[17]. To explore if mitosin was also subjected to similar translocation, we performed the same ATP inhibitor assay in CV1 cells. As shown in Fig 4A, treatment with azide resulted in significant spindle pole accumulation of mitosin in cells at prometaphase (panels 1-2) and metaphase (panels 34). In contrast, mitosin still predominantly bound to centromeres in mock-treated cells (Fig 4B, panels 14). To further confirm that such translocation was exerted by dynein, we utilized a dominant negative mutant, Nudel ${ }^{\mathrm{N} 20}$, which, when overexpressed, impairs the dynein function in M phase[25]. As a control, GFP-Nudel and mitosin were translocated to spindle poles after azide treatment (Fig 4C, panels 1-3). In GFP-Nudel $^{\mathrm{N} 20}$ expressors, however, neither the N20 mutant nor mitosin was fully tranported to the spindle

poles (Fig 4C, panels 4-6). Such patterns were consis-

A
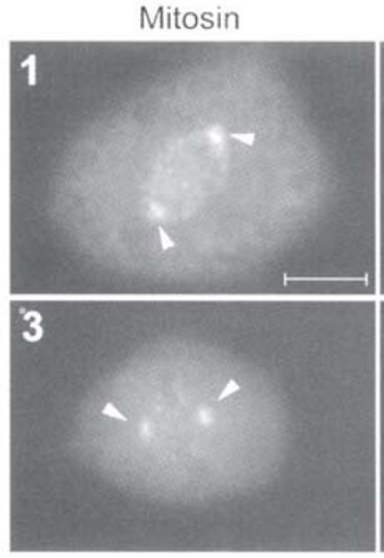

B
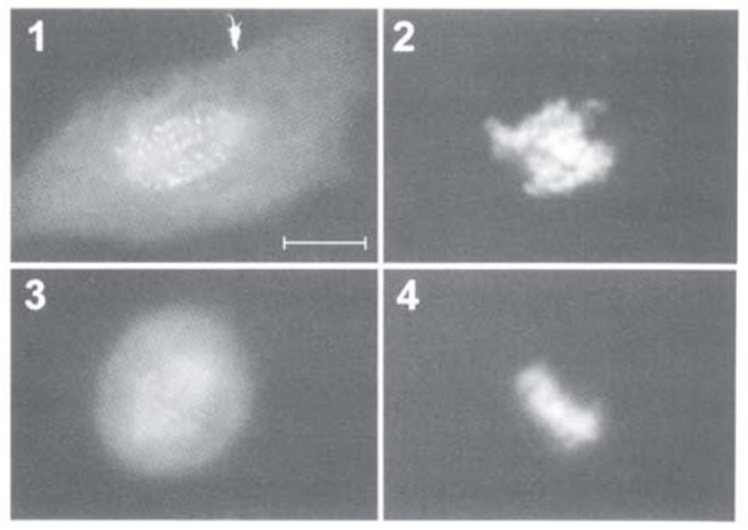

C

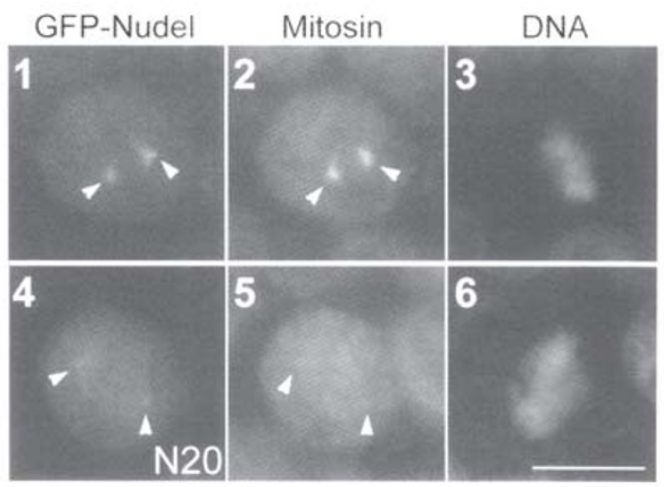

Fig 4. Translocation of mitosin from kinetochores to spindle poles by cytoplasmic dynein. CV1 cells were treated with Naazide and 2-deoxyglucose (A) or just buffer (B) for $30 \mathrm{~min}$ at $37^{\circ} \mathrm{C}$ before fixation and IIF labeling with anti-mitosin mAb. Typical prometaphase cells (panels 1-2) and metaphase cells (panels 3-4) are shown. Spindle poles are indicated by arrowheads. In (C), a typical mitotic HEK293T cell expressing GFP-Nudel (panels 1-3) or GFP-Nudel ${ }^{\mathrm{N} 20}$ (panels 4-6) after the azide treatment was shown. Scale bar $=10 \mu \mathrm{m}$. 
tent with our previous report[25].

\section{Cloning of the duplicated region of mitosin gene from eutherian mammals}

Considering the conservation of the core region from chicken to human (Fig 2A),it is likely that at least mitosin orthologs containing the repeat region will be targeted to kinetochores. According to the kinetochore staining in mouse, dog, and mink cells (Fig 2B), we speculated that probably all mammalian mitosin orthologs contained the repeat region. Since this repeat is located in one exon in human, we performed PCR with a pair of degenerate primers, using genomic DNA as templates. Theoretically, DNA fragments coding for either one repeat unit (419 bp) or both repeat units (972 bp) should exist in the PCR products. The longer fragment was successfully cloned from canine genome (GenBank accession AF182408), while two types of closely-related short fragments were cloned from rabbit, each representing a repeat unit (GenBank accessions AF182410S1 and AF182410S2). Existence of the internal repeat region in canine mitosin is in agreement with our speculation.

We then tried to estimate possible existence of the repeat region in other mammals via an evolution analysis. From the evolution point of view, it is believed that closely related DNA fragments usually originated from a common ancestor[20]. The separation time for any two of such sequences can be estimated when the divergence rate, i.e., the percent of sequence difference per million years, for the given group is known. Moreover, the divergence calculated from replacement sites, i.e., sites at which a mutation alters the encoded amino acid, is relatively proportional to the separation time of the two sequences[20]. We therefore analyzed the divergence between repeat units of mitosin orthologs. The divergences of mammalian mitosin varied from $7.1 \%$ to $12.5 \%$ between species, and from $24.4 \%$ to $30.5 \%$ compared with CMF1. In contrast, within a mammalian species, the two repeat units exhibited an average divergence of $17.81 .5 \%$. It is believed that Primate (human), Lagomorpha (rabbit), and Carnivora (dog) diverged from each other at approximately 91-92 million years ago (MYA) in evolution[26], while Aves (chicken) separated from Mammalia at 310 MYA[26,27]. A correlation between divergence and separation time can then be established by linear regression ( (Fig 5).). The average divergence between the first and second repeat units therefore corresponded to a separation time of 19317 MYA (Fig 5). Similar result (2021 MYA) was obtained when the divergence rate was calculated as the replacement rate of amino acids. Such a time is even earlier than separation of marsupials from placental mammals at $\sim 173$ MYA[26], although it remains to be verified that marsupial mitosin also contain the repeat. Fixation of such duplication in mammalian

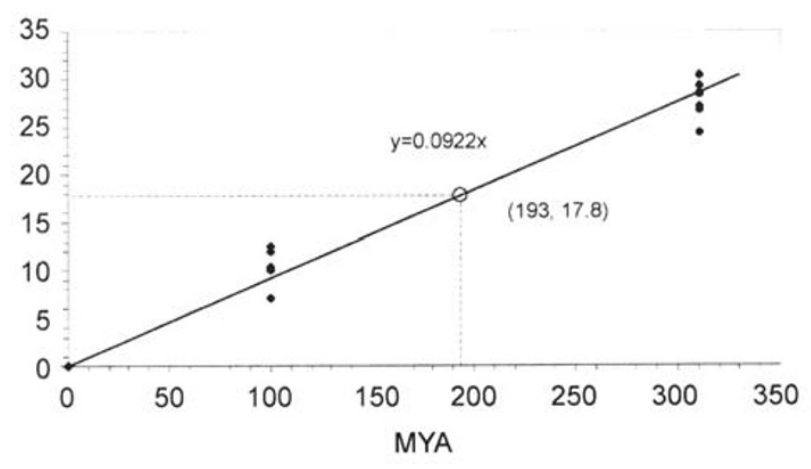

Fig 5. The divergence rate of mitosin's repeat region. Correlation between divergence and time is established by linear regression by assuming that the divergence before separation of two species is $0 \%$. Accordingly, a divergence of $17.8 \pm 1.5 \%$ between the first and second repeat units of mammalian mitosin corresponds to a separation time of $193 \pm 17$ MYA.

genomes implies importance of this event for successful evolution of mammals.

\section{DISCUSSION}

Several lines of evidence suggest that human mitosin and at least its eutherian orthologs are intrinsic kinetochore proteins. First, the centromere localization of mitosin in primary cells (Fig 1) indicates that such a property does not depend on cell transformation as suspected[9]. Second, when expressed in Chinese hamster and rat cells, mitosin confers centromere localization $[5,18]$, indicating existence of mitosin target (s) on the kinetochore in these rodent cells. Third, mitosin orthologs in murine, canine, and mink cells also exhibit centromere localization (Fig 2). We confirmed that exogenous LEK1 also located to the centromere (Fig 2D). Recognition of LEK1C by the antimitosin IgY (Fig 2C) further indicates that the centromere staining in NIH3T3 by the same antibody (Fig 2B, panels 1-3) was due to LEK1. Therefore, the 
anti-LEK1 antibodies by Goodwin et al[9] might not be appropriate for IIF of the mitotic LEK1 due to, for instance, failure to recognize phosphorylated LEK1. Forth, we provided evidence to support that the internal repeat of mitosin important for its strong centromere localization[18] is conserved in all placental mammals (Fig 4), since duplication of the repeat unit occurred at around 193 MYA, a time apparently after separation of the common ancestor of avians and mammals[26]. Since the core region, another domain for strong kinetochore localization, is conserved in chicken and mouse (Fig 2A), placental mitosin orthologs are very likely to be all kinetochore proteins.

Our findings further imply a role of mitosin in the spindle checkpoint during $\mathrm{M}$ phase. Kinetochore proteins involved in the spindle checkpoint are located at the outer kinetochore plate or corona and exhibit reduced binding to kinetochores after microtubule attachment to the latter[12, 22, 24]. It has recently been shown that checkpoint proteins, but not structural proteins at the outer kinetochore, are constitutively transported from kinetochores to the spindle poles in microtubule dependent manner by dynein/dynactin complex during mitosis[17]. Such a translocation process contributes to inactivation of the spindle checkpoint, but is dispensable for both chromosome congression during prometaphase/metaphase transition and chromosome segregation in anaphase[17]. Mitoisn is also an outer kinetochore protein[2, 4]. Enhanced binding of mitosin to unaligned chromosomes (Fig 3) suggests that the kinetochore localization of mitosin is also sensitive to microtubule attachment. It was also subjected to similar poleward translocation by dynein. These properties imply that mitosin might also function either directly or indirectly in the spindle checkpoint. In addition, such translocation also provided an explanation for the bizarre distribution of mitosin (and many other checkpoint proteins) at kinetochores, spindle poles, and in cytoplasm (Fig 4)[1, 5]: in mitotic cells, mitosin in the cytoplasm may continuously bind to the kinetochore and is then transported to spindle poles along microtubules. Taken together, mitosin/LEK1/CMF1 family may serve as multifunctional proteins involved in both mitosis and differentiation.

\section{ACKNOWLEDGEMENTS}

The authors want to thank Qiong Ping HUANG and Kong Hua ZHANG for technical assistance; Yi Dong SHEN and Yong Li SHAN for proofreading the manuscript. We are also in debt to colleagues for generously providing HUVEC (Jian Guo GENG), Lak cells (Zhi Jiang WU), MDCK, and Mv 1 Lu (Jian Guo SONG). This work was supported by grants 97JC14006 from Shanghai Committee of Science and Technology, (No. 30025021, 39970160, and 39500030) from the Natural Science Foundation of China, and KSCX2-2-02 from Chinese Academy of Sciences.

\section{REFERENCES}

1. Zhu X, Mancini MA, Chang KH et al. Characterization of a novel 350-kilodalton nuclear phosphoprotein that is specifically involved in mitotic-phase progression. Mol Cell Biol 1995; 15:5017-29.

2. Zhu X, Chang KH, He D, Mancini MA, Brinkley WR, Lee WH. The C-terminus of mitosin is essential for its nuclear localization, centromere/kinetochore targeting, and dimerization. J Biol Chem 1995; 270:19545-50.

3. Liao H, Winkfein RJ, Mack G, Rattner JB and Yen TJ. CENP$\mathrm{F}$ is a protein of the nuclear matrix that assembles onto kinetochores at late G2 and is rapidly degraded after mitosis. J cell Biol 1995; 130:507-18.

4. Rattner JB, Rao A, Fritzler MJ, Valencia DW, Yen TJ. CENP$\mathrm{F}$ is a ca. $400 \mathrm{KDa}$ kinetochore protein that exhibits a cellcycle dependent localization. Cell Motil Cytoskeleton 1993; 26:214-26.

5. Zhu X, Ding L, Pei G. Carboxyl terminus of mitosin is sufficient to confer spindle pole localization. J Cell Biochem 1997; 66:441-9.

6. Ashar HR, James L, Gray K, et al. Farnesyl transferase inhibitors block the farnesylation of CENP-E and CENP-F and alter the association of CENP-E with the microtubules. J Biol Chem 2000; 275:30451-7.

7. Hussein D, Taylor SS. Farnesylation of Cenp-F is required for G2/M progression and degradation after mitosis. J Cell Sci 2002; 115:3403-14.

8. Wei Y, Bader D, Litvin J. Identification of anovel cardiacspecific transcript critical for cardiac myocyte differentiation. Development 1996; 122:2779-89.

9. Goodwin RL, Pabon-Pena LM, Foster GC, Bader D. The cloning and analysis of LEK1 Identifies Variations in the LEK/ Centromere Protein F/Mitosin Gene Family. J Biol Chem 1999; 274:18597-604.

10. Pabon-Pena LM, Goodwin RL, Cise LJ, Bader D. Analysis of CMF1 reveals a bone morphogenetic protein-independent component of the cardiomyogenic pathway. J Biol Chem 2000; 275:21453-9.

11. Dees E, Pabon-Pena LM, Goodwin RL, Bader D. Characterization of CMF1 in avian skeletal muscle. Dev Dyn 2000; 219:169-81.

12. Shah JV, Cleveland DW. Waiting for anaphase: Mad2 and 
the spindle assembly checkpoint. Cell 2000; 103:997-1000.

13. Musacchio A, Hardwick KG. The spindle checkpoint: structural insights into dynamic signalling. Nat Rev Mol Cell Biol 2002; 3:731-41

14. Gassmann M, Th鰉mes P, Weiser T, Hubscher U. Efficient production of chicken egg yolk antibodies against a conserved mammalian protein. FASEB J 1990; 4:2528-32.

15. Geng JG, Bevilacqua MP, Moore KL, et al. Rapid neutrophil adhesion to activated endothelium mediated by GMP140. Nature 1990; 343:757-60.

16. Howell BJ, Hoffman DB, Fang G, Murray AW, Salmon ED. Visualization of Mad2 dynamics at kinetochores, along spindle fibers, and at spindle poles in living cells. J Cell Boil 2000; 150:1233-50.

17. Howell BJ, McEwen BF, Canman JC, et al. Cytoplasmic dynein/dynactin drives kinetochore protein transport to the spindle poles and has a role in mitotic spindle checkpoint inactivation. J Cell Biol 2001; 155:1159-72.

18. Zhu X. Structural requirements and dynamics of mitosinkinetochore interaction in M phase. Mol Cell Biol 1999; 19: 1016-24.

19. Chou Q, Russell M, Birch DE, Raymond J, Bloch W. Prevention of pre-PCR mis-priming and primer dimerization improves low-copy-number amplifications. Nucleic Acids Res 1992; 20:1717-23.
20. Lewin B. Genes V 1994; Oxford University Press and Cell Press. Dobie KW, Hari KL, Maggert KA, Karpen GH. Centromere proteins and chromosome inheritance: a complex affair. Curr Opin Genet 1999; 9:206-17.

21. Maney T, Ginkel LM, Hunter AW, Wordeman L. The kinetochore of higher eukaryotes: a molecular view. Int Rev Cytol 1999; 194:67-131.

22. King JM, Hays TS, Nicklas RB. Dynein is a transient kinetochore component whose binding is regulated by microtubule attachment, not tension. J Cell Biol 2000; 151:73948.

23. Hoffman DB, Pearson CG, Yen TJ, Howell BJ, Salmon ED. Microtubule dependent changes in the assembly of microtubule motor proteins and mitotic spindle checkpoint proteins at PtK1 kientochores. Mol Biol Cell 2001; 12:19952009.

24. Yan X, Li F, Liang Y, et al. Human Nudel and NudE as Regulators of Cytoplasmic Dynein in Poleward Protein Transport along the Mitotic Spindle. Mol Cell Biol 2003; 23:1239-50.

25. Kumar S, Hedges SB. A molecular timescale for vertebrate evolution. Nature 1998; 392:917-20.

26. Novacek MJ. Mammalian phylogeny: shaking the tree. Nature 1992; 356:121-5. 